\title{
Virus shapes and buckling transitions in spherical shells*
}

\author{
DAVID R. NELSON \\ Lyman Laboratory of Physics, Harvard University, Cambridge, MA 02138, USA
}

We show that the icosahedral packings of protein capsomeres proposed by Caspar and Klug for spherical viruses become unstable to faceting for sufficiently large virus size, in analogy with the buckling instability of disclinations in two-dimensional crystals. The faceted shape depends only on the dimensionless Foppl-von Karman number $\gamma=Y R^{2} / \kappa$, where $Y$ is the two-dimensional Young's modulus of the protein shell, $\gamma$ is its bending rigidity and $R$ is the mean virus radius. Our model, based on the nonlinear physics of thin elastic shells, produces excellent one parameter fits in real space to the full threedimensional shape of large spherical viruses such as HK97 $(\gamma=1480)$ and Yeast L-A $(\gamma=547)$. The shape can be parameterized more quantitatively in terms of a spherical harmonic expansion. We also investigate elastic shell theory for extremely large $\gamma, 10^{3}<\gamma<10^{8}$, and find results applicable to icosahedral shapes of large vesicles studied with freeze fracture and electron microscopy. Finally, we discuss an instability towards grain boundaries in shells with an exceptionally large bending rigidity, which has been observed experimentally in related problems in spherical crystallography such as the packing of colloidal particles on the surface of water droplets in oil [1].

\section{References}

[1] Bausch, A.R., Bowick, M.J., Cacciuto, A., Dinsmore, A.D., Hsu, M.F., Nelson, D.R., Nikolaides, M.G., Travesset, A. and Weitz, A., 2003, Grain boundary scars and spherical crystallography. Science, 299, 1716.

*Work on viruses carried out in collaboration with Jack Lidmar and Leonid Mirny: More information can be found in Phys. Rev. E 68, 051910 (2003) or http://www.arxiv.org/PS_cache/cond-mat/pdf/0306/0306741.pdf 


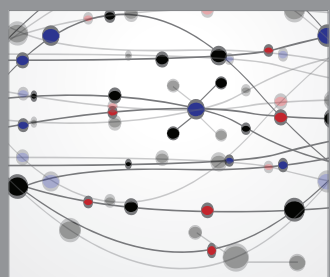

The Scientific World Journal
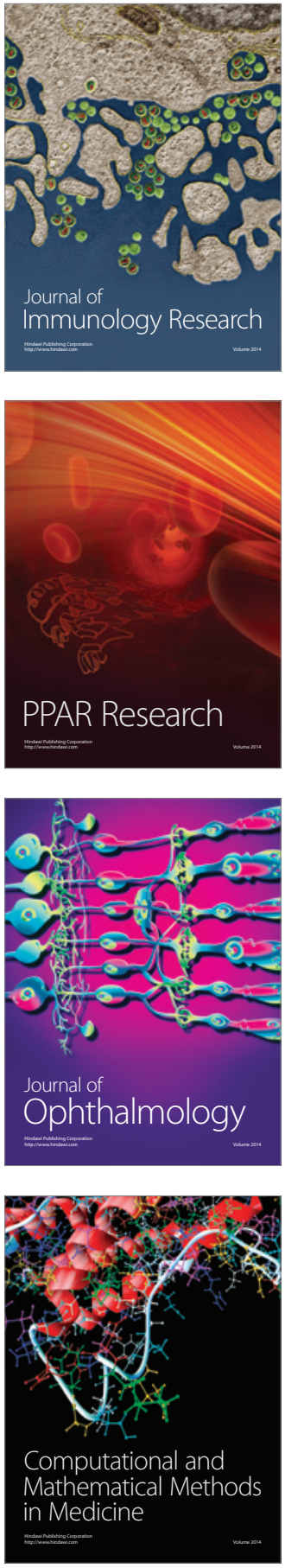

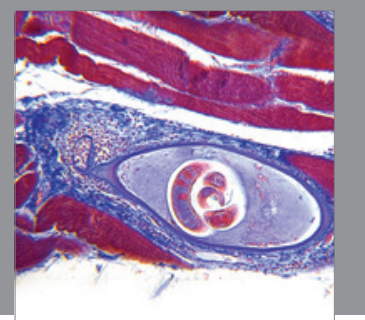

Gastroenterology

Research and Practice
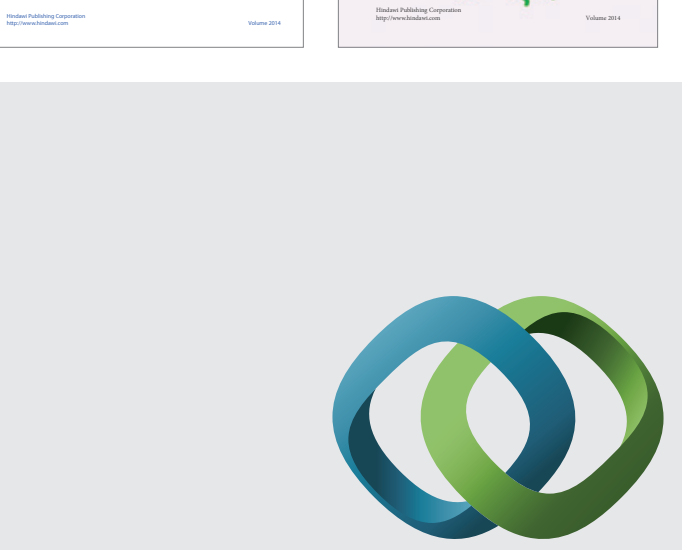

\section{Hindawi}

Submit your manuscripts at

http://www.hindawi.com
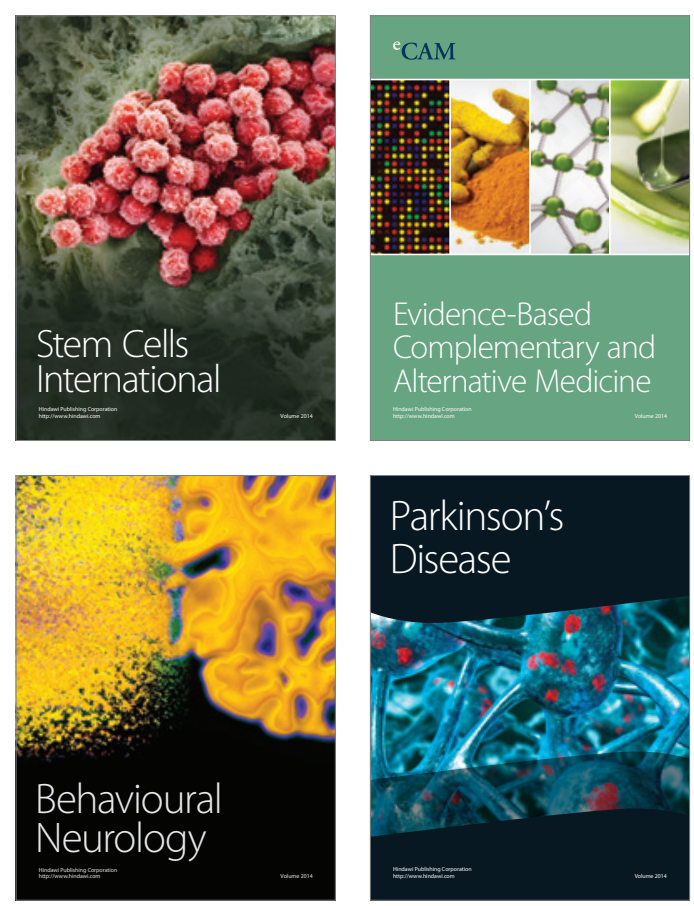

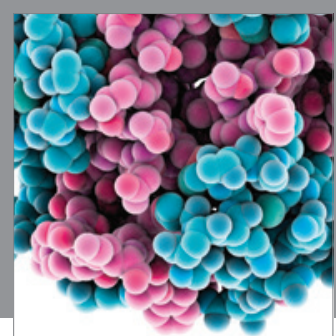

Journal of
Diabetes Research

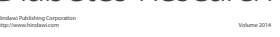

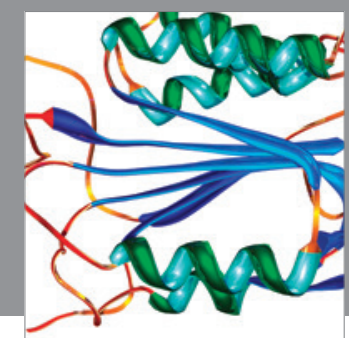

Disease Markers
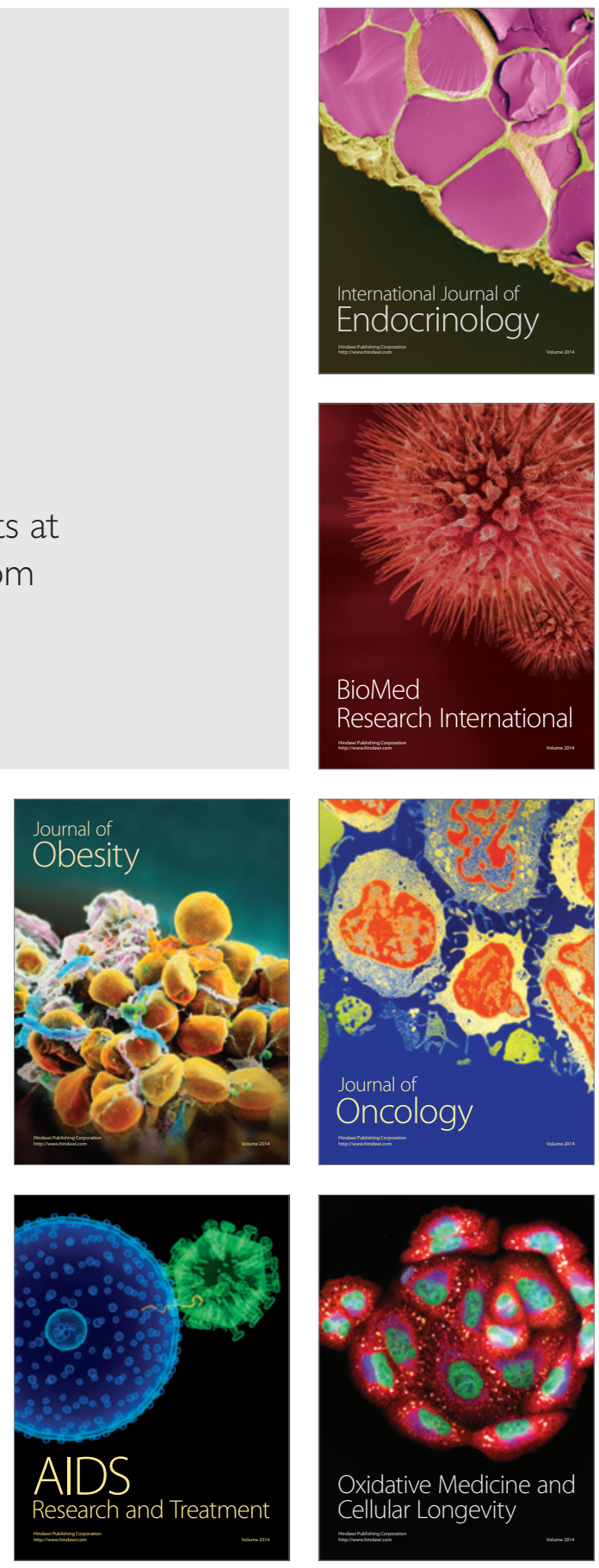